\title{
Order-Value Optimization: Formulation and Solution by means of a Primal Cauchy Method
}

\author{
Roberto Andreani* $\quad$ Cibele Dunder ${ }^{\dagger} \quad$ José Mario Martínez ${ }^{\ddagger}$
}

July 21, 2004

\begin{abstract}
The Order-Value Optimization (OVO) problem is a generalization of the classical Minimax problem. Instead of the maximum of a set functions, the functional value that ranks in the $p$-th place is minimized. The problem seeks the application to (non-pessimistic) decision making and to model fitting in the presence of (perhaps systematic) outliers. A Cauchy-type method is introduced that solves the problem in the sense that every limit point satisfies an adequate optimality condition. Numerical examples are given.

Key words: Order-Value Optimization, iterative methods, global convergence, fitting parameters.
\end{abstract}

\section{Introduction}

Given $m$ functions $f_{1}, \ldots, f_{m}$, defined in a domain $\Omega \subset \mathbb{R}^{n}$ and an integer $p \in\{1, \ldots, m\}$, the $(p-)$ Order-Value (OVO) function $f$ is given by

$$
f(x)=f_{i_{p}(x)}(x)
$$

${ }^{*}$ Department of Applied Mathematics, IMECC-UNICAMP, University of Campinas, CP 6065, 13081-970 Campinas SP, Brazil. This author was supported by FAPESP (Grant 01/05492-1) and CNPq (Grant 301115/96-6). E-Mail: andreani@ime.unicamp.br.

${ }^{\dagger}$ Department of Applied Mathematics, IME-USP, University of Sao Paulo, CEP 05508900 Sao Paulo, SP, Brazil. E-mail: dunder@ime.usp.br

${ }^{\ddagger}$ Department of Applied Mathematics, IMECC-UNICAMP, University of Campinas, CP 6065, 13081-970 Campinas SP, Brazil. This author was supported by PRONEX, FAPESP (PT 2001-04597-4) E-Mail: martinez@ime.unicamp.br 
for all $x \in \Omega$, where

$$
f_{i_{1}(x)}(x) \leq f_{i_{2}(x)}(x) \leq \ldots \leq f_{i_{p}(x)}(x) \leq \ldots \leq f_{i_{m}(x)}(x) .
$$

If $p=1, f(x)=\min \left\{f_{1}(x), \ldots, f_{m}(x)\right\}$ whereas for $p=m$ we have that $f(x)=\max \left\{f_{1}(x), \ldots, f_{m}(x)\right\}$.

We will show later that $f$ is continuous. However, even if the functions $f_{i}$ are differentiable, the OVO function is not smooth.

The OVO problem consists in the minimization of the Order-Value function:

$$
\text { Minimize } f(x) \text { s.t. } x \in \Omega \text {. }
$$

The definition of the OVO problem was motivated by two main applications.

(1) Assume that $\Omega$ is a space of decisions and, for each $x \in \Omega, f_{i}(x)$ represents the cost of decision $x$ under the scenario $i$. The Minimax decision corresponds to choose $x$ in such a way that the maximum possible cost is minimized. This is a very pessimistic alternative and decision-makers usually prefer to discard the worst possibilities in order to proceed in a more realistic way. For example, the decision maker may want to discard the $10 \%$ more pessimistic scenarios. This corresponds to minimize the $p$-Order-Value function with $p \approx 0.9 \mathrm{~m}$.

(2) Assume that we have a parameter-estimation problem where the space of parameters is $\Omega$ and $f_{i}(x)$ is the error in the observation $i$ when we adopt the parameter $x$. The Minimax estimation problem corresponds to minimize the maximum error. As it is well-known this estimate is very sensitive to the presence of outliers. Many times, we want to eliminate (say) the $15 \%$ larger errors because the can represent wrong observations. This corresponds to minimize the $p$-Order-Value function with $p \approx 0.85 \times m$. The OVO strategy is especially designed to eliminate the influence of systematic errors.

In this paper we introduce a steepest descent type method for solving (1). We say that this method is "primal" because no auxiliar or dual variables are involved in it. The algorithm is presented in Section 2, where a convergence theorem is also given. In Section 3 we describe a way to solve approximately the subproblems, satisfying the theoretical requirements. Numerical examples are shown in Section 4. Conclusions are given in the last section of this paper. 


\section{The algorithm}

Assume that $\Omega \subset \mathbb{R}^{n}$ is closed and convex, and $f_{1}, \ldots, f_{m}$ have continuous partial derivatives in an open set that contains $\Omega$. (The fact that $\Omega$ is closed and convex will be used in the optimality condition, in the definition of the subproblems of the main algorithm and in the proofs of Theorems 2.2 and 2.3 below.) We denote $g_{j}=\nabla f_{j}$ from now on.

For all $x, y \in \Omega, j=1, \ldots, m$, we assume that

$$
\left\|g_{j}(x)\right\|_{\infty} \leq c,
$$

and

$$
\left\|g_{j}(y)-g_{j}(x)\right\|_{\infty} \leq L\|y-x\|_{\infty} .
$$

Consequently, for all $x, y \in \Omega, j=1, \ldots, m$,

$$
\left|f_{j}(y)-f_{j}(x)\right| \leq c\|y-x\|_{\infty}
$$

and

$$
f_{j}(y) \leq f_{j}(x)+g_{j}(x)^{T}(y-x)+\frac{L}{2}\|y-x\|_{\infty}^{2} .
$$

Given $\varepsilon \geq 0, x \in \Omega$, we define

$$
I_{\varepsilon}(x)=\left\{j \in\{1, \ldots, m\} \mid f(x)-\varepsilon \leq f_{j}(x) \leq f(x)+\varepsilon\right\} .
$$

The following theorem shows the continuity of the OVO-function.

Theorem 2.1. The p-order-value function $f$ is continuous.

Proof. Assume that $x^{k} \rightarrow x$, and suppose that, for all $k$ in some subsequence,

$$
\left|f\left(x^{k}\right)-f(x)\right| \geq \delta>0 .
$$

For that subsequence, there exists an index $j \in\{1, \ldots, m\}$ such that

$$
f\left(x^{k}\right)=f_{j}\left(x^{k}\right)
$$

infinitely many times. Therefore,

$$
f_{j}\left(x^{k}\right) \geq f_{\ell}\left(x^{k}\right)
$$

for at least $p$ indices $\ell \in\{1, \ldots, m\}$. Moreover,

$$
f_{j}\left(x^{k}\right) \leq f_{\ell}\left(x^{k}\right)
$$


for at least $m-p+1$ indices $\ell$ in the set $\{1, \ldots, m\}$. Since the number of subsets of $\{1, \ldots, m\}$ is finite, a set of indices $\ell$ that verify (6) is repeated infinitely many times, and the same happens with a set of indices $\ell$ that verify (7). Therefore, taking limits in (6) and (7), we obtain that

$$
f_{j}(x) \geq f_{\ell}(x)
$$

for at least $p$ indices $\ell \in\{1, \ldots, m\}$ and

$$
f_{j}(x) \leq f_{\ell}(x)
$$

for at least $m-p+1$ indices $\ell \in\{1, \ldots, m\}$. Therefore,

$$
f(x)=f_{j}(x)
$$

But $f_{j}\left(x^{k}\right) \rightarrow f_{j}(x)$, so this contradicts (5).

The theorem below provides a necessary optimality condition for the OVO problem.

Definition. We say that $x$ is $\varepsilon-$ optimal if

$$
\mathcal{D} \equiv\left\{d \in \mathbb{R}^{n} \mid x+d \in \Omega \text { and } g_{j}(x)^{T} d<0 \quad \forall j \in I_{\varepsilon}(x)\right\}=\emptyset .
$$

Theorem 2.2. If $x_{*} \in \Omega$ is a local minimizer of $f(x)$ subject to $x \in \Omega$ and $\varepsilon \geq 0$, then $x_{*}$ is $\varepsilon-$ optimal.

Proof. Suppose, by contradiction, that (8) is not true. Then, there exists $d \in \mathbb{R}^{n}$ and $\bar{\alpha}>0$ such that $x_{*}+d \in \Omega$ and

$$
f_{j}\left(x_{*}+\alpha d\right)<f_{j}\left(x_{*}\right) \forall \alpha \in(0, \bar{\alpha}]
$$

for all $j \in I_{\varepsilon}\left(x_{*}\right)$.

So, for all $j \in\{1, \ldots, m\}$ with $f_{j}\left(x_{*}\right)=f\left(x_{*}\right)$, one has that $j \in I_{\varepsilon}\left(x_{*}\right)$ and then one gets, under the supposition of the proof, that (9) holds.

Define

$$
\begin{aligned}
& \varepsilon_{1}=\min _{f_{j}\left(x_{*}\right)<f\left(x_{*}\right)}\left\{f\left(x_{*}\right)-f_{j}\left(x_{*}\right)\right\} \in(0, \infty], \\
& \varepsilon_{2}=\min _{f_{j}\left(x_{*}\right)>f\left(x_{*}\right)}\left\{f_{j}\left(x_{*}\right)-f\left(x_{*}\right)\right\} \in(0, \infty],
\end{aligned}
$$


Let $\tilde{\alpha} \leq \bar{\alpha}$ be such that

$$
\left|f_{j}\left(x_{*}+\alpha d\right)-f_{j}\left(x_{*}\right)\right|<\frac{\min \left\{\varepsilon_{1}, \varepsilon_{2}\right\}}{2}
$$

for all $j=1, \ldots, m, \alpha \in(0, \tilde{\alpha}]$.

Therefore, for all $\alpha \in(0, \tilde{\alpha}]$, the index $j$ such that

$$
f\left(x_{*}+\alpha d\right)=f_{j}\left(x_{*}+\alpha d\right)
$$

is one of the indices $j$ such that $f\left(x_{*}\right)=f_{j}\left(x_{*}\right)$. In other words, this index belongs to $I_{0}\left(x_{*}\right) \subset I_{\varepsilon}\left(x_{*}\right)$. But, by $(9)$,

$$
f_{j}\left(x_{*}+\alpha d\right)<f_{j}\left(x_{*}\right)
$$

for all $\alpha \in(0, \tilde{\alpha}]$. Therefore, $x_{*}$ is not a local minimizer.

Below we describe the main algorithm. It is a primal method in the sense that only primal variables $x$ are manipulated and updated at each iteration. The algorithm finds a decreasing sequence of functional values using search directions that come from the (inexact) resolution of a convex programming problem.

\section{Algorithm 2.1}

Let $x_{0} \in \Omega$ an arbitrary initial point. Let $\theta \in(0,1), \Delta>0, \varepsilon>0$, $0<\sigma_{\min }<\sigma_{\max }<1, \eta \in(0,1]$.

Given $x_{k} \in \Omega$ the steps of the $k$-th iteration are:

Step 1. (Solving the subproblem)

Define

$$
M_{k}(d)=\max _{j \in I_{\varepsilon}\left(x_{k}\right)} g_{j}\left(x_{k}\right)^{T} d .
$$

Consider the subproblem

$$
\text { Minimize } M_{k}(d) \text { s.t. } x_{k}+d \in \Omega,\|d\|_{\infty} \leq \Delta .
$$

Note that (11) is equivalent to the convex optimization problem

Minimize $w$

$$
g_{j}\left(x_{k}\right)^{T} d \leq w \forall j \in I_{\varepsilon}\left(x_{k}\right),
$$




$$
x_{k}+d \in \Omega, \quad\|d\|_{\infty} \leq \Delta .
$$

Let $\bar{d}_{k}$ be a solution of (11). (We will see later that we do not need to compute it.) Let $d_{k}$ be such that $x_{k}+d_{k} \in \Omega,\left\|d_{k}\right\| \leq \Delta$ and

$$
M_{k}\left(d_{k}\right) \leq \eta M_{k}\left(\bar{d}_{k}\right)
$$

If $M_{k}\left(d_{k}\right)=0$ stop.

Step 2. (Steplength calculation)

Set $\alpha \leftarrow 1$.

If

$$
f\left(x_{k}+\alpha d_{k}\right) \leq f\left(x_{k}\right)+\theta \alpha M_{k}\left(d_{k}\right)
$$

set $\alpha_{k}=\alpha, x_{k+1}=x_{k}+\alpha_{k} d_{k}$ and finish the iteration. Otherwise, choose $\alpha_{\text {new }} \in\left[\sigma_{\min } \alpha, \sigma_{\max } \alpha\right]$, set $\alpha \leftarrow \alpha_{\text {new }}$ and repeat the test (13).

The following is a technical lemma which will be useful in the convergence proof.

Lemma 2.1. Assume that $a_{1}, \ldots, a_{r}$ are real numbers such that

$$
a_{1} \leq a_{2} \leq \ldots \leq a_{q} \leq \ldots \leq a_{r}
$$

Suppose that $\beta>0$ and $b_{1}, \ldots, b_{r} \in \mathbb{R}$ are such that

$$
b_{j} \leq a_{j}-\beta \forall j=1, \ldots, r
$$

and

$$
b_{i_{1}} \leq b_{i_{2}} \leq \ldots \leq b_{i_{q}} \leq \ldots \leq b_{i_{r}} .
$$

Then,

$$
b_{i_{q}} \leq a_{q}-\beta
$$

Proof. Clearly,

$$
b_{i_{q}} \leq a_{i_{q}}-\beta, b_{i_{q}} \leq b_{i_{q+1}} \leq a_{i_{q+1}}-\beta, \ldots, b_{i_{q}} \leq b_{i_{r}} \leq a_{i_{r}}-\beta .
$$

Therefore,

$$
b_{i_{q}} \leq \min \left\{a_{i_{q}}, \ldots, a_{i_{r}}\right\}-\beta .
$$

But, since $a_{1} \leq \ldots \leq a_{q} \leq \ldots \leq a_{r}$, we have that

$$
\min \left\{a_{i_{q}}, \ldots, a_{i_{r}}\right\} \leq a_{q} .
$$


Therefore,

$$
b_{i_{q}} \leq a_{q}-\beta
$$

as we wanted to prove.

In the following theorem, we state that, if the iterate $x_{k}$ is not $\varepsilon$-optimal, then $x_{k+1}$ is well defined and $\alpha_{k}$ is bounded away from zero.

Theorem 2.3. Assume that $x_{k} \in \Omega$ is the $k$-th iterate of Algorithm 2.1. Then:

(a) The algorithm stops at $x_{k}$ if, and only if, $x_{k}$ is $\varepsilon$ - optimal.

(b) If the algorithm does not stop at $x_{k}$, then the iteration is well-defined and

$$
\alpha_{k} \geq \min \left\{\frac{2 \sigma_{\min } \gamma_{k}(1-\theta)}{L \Delta^{2}}, \frac{\varepsilon \sigma_{\min }}{3 c \Delta}\right\}
$$

where

$$
\gamma_{k}=-\max _{j \in I_{\varepsilon}\left(x_{k}\right)}\left\{g_{j}\left(x_{k}\right)^{T} d_{k}\right\}>0 .
$$

Proof. If the algorithm stops at $x_{k}$, then $M_{k}\left(d_{k}\right)=0$. Therefore, by (12), $M_{k}\left(\bar{d}_{k}\right)=0$. So, $M_{k}(d) \geq 0$ for all $d \in \mathcal{D}$ such that $\|d\|_{\infty} \leq \Delta$. Thus, $M_{k}(d) \geq 0$ for all $d \in \mathcal{D}$. This implies that $x_{k}$ is $\varepsilon$-optimal.

Reciprocally, if $x_{k}$ is $\varepsilon$-optimal, we must have that $M_{k}\left(\bar{d}_{k}\right)=0$, so $M_{k}\left(d_{k}\right)=0$ and the algorithm stops at $x_{k}$.

If the algorithm does not stop at $x_{k}$, then $M_{k}\left(d_{k}\right)<0$. Therefore,

$$
-\gamma_{k}=\max _{j \in I_{\varepsilon}\left(x_{k}\right)}\left\{g_{j}\left(x_{k}\right)^{T} d_{k}\right\}<0 .
$$

Assume that

$$
\alpha \in\left[0, \frac{2 \gamma_{k}(1-\theta)}{L \Delta^{2}}\right]
$$

Then,

$$
\frac{L \alpha \Delta^{2}}{2} \leq(1-\theta) \gamma_{k}
$$

So,

$$
\frac{L \alpha \Delta^{2}}{2} \leq(\theta-1) g_{j}\left(x_{k}\right)^{T} d_{k} \forall j \in I_{\varepsilon}\left(x_{k}\right) .
$$

Therefore,

$$
g_{j}\left(x_{k}\right)^{T} d_{k}+\frac{L \alpha \Delta^{2}}{2} \leq \theta g_{j}\left(x_{k}\right)^{T} d_{k} \quad \forall j \in I_{\varepsilon}\left(x_{k}\right) .
$$


Therefore, since $\left\|\alpha d_{k}\right\|_{\infty} \leq \Delta$,

$$
\alpha g_{j}\left(x_{k}\right)^{T} d_{k}+\frac{L \alpha^{2}\left\|d_{k}\right\|_{\infty}^{2}}{2} \leq \alpha \theta g_{j}\left(x_{k}\right)^{T} d_{k} \forall j \in I_{\varepsilon}\left(x_{k}\right) .
$$

So,

$$
f_{j}\left(x_{k}\right)+g_{j}\left(x_{k}\right)^{T}\left(\alpha d_{k}\right)+\frac{L}{2}\left\|\alpha d_{k}\right\|_{\infty}^{2} \leq f_{j}\left(x_{k}\right)+\alpha \theta g_{j}\left(x_{k}\right)^{T} d_{k} \forall j \in I_{\varepsilon}\left(x_{k}\right) .
$$

Therefore, by (3)

$$
f_{j}\left(x_{k}+\alpha d_{k}\right) \leq f_{j}\left(x_{k}\right)+\alpha \theta g_{j}\left(x_{k}\right)^{T} d_{k} \forall j \in I_{\varepsilon}\left(x_{k}\right) .
$$

So, we have proved that, if $\alpha \in\left[0,2 \gamma_{k}(1-\theta) /\left(L \Delta^{2}\right)\right]$,

$$
f_{j}\left(x_{k}+\alpha d_{k}\right) \leq f_{j}\left(x_{k}\right)+\alpha \theta M_{k}\left(d_{k}\right) \forall j \in I_{\varepsilon}\left(x_{k}\right) .
$$

On the other hand, if $\alpha \in[0, \varepsilon /(3 c \Delta)]$, we have that $\alpha c \Delta \leq \varepsilon / 3$, so $\alpha c\left\|d_{k}\right\|_{\infty} \leq \varepsilon / 3$, so $c\left\|\alpha d_{k}\right\|_{\infty} \leq \varepsilon / 3$, therefore, by (2),

$$
\left|f_{j}\left(x_{k}+\alpha d_{k}\right)-f_{j}\left(x_{k}\right)\right| \leq \frac{\varepsilon}{3} \forall j=1, \ldots, m .
$$

Therefore, for all $\ell=1, \ldots, p$,

$$
f_{i_{\ell}\left(x_{k}\right)}\left(x_{k}+\alpha d_{k}\right) \leq f_{i_{\ell}\left(x_{k}\right)}\left(x_{k}\right)+\frac{\varepsilon}{3} \leq f\left(x_{k}\right)+\frac{\varepsilon}{3}
$$

and, for all $\ell=p, \ldots, m$,

$$
f_{i_{\ell}\left(x_{k}\right)}\left(x_{k}+\alpha d_{k}\right) \geq f_{i_{\ell}\left(x_{k}\right)}\left(x_{k}\right)-\frac{\varepsilon}{3} \geq f\left(x_{k}\right)-\frac{\varepsilon}{3} .
$$

This means that at least $p$ elements of the set

$$
\left\{f_{1}\left(x_{k}+\alpha d_{k}\right), \ldots, f_{m}\left(x_{k}+\alpha d_{k}\right)\right\}
$$

are less than or equal to $f\left(x_{k}\right)+\varepsilon / 3$ and that at least $m-p+1$ elements of that set are greater than or equal to $f\left(x_{k}\right)-\varepsilon / 3$.

Therefore,

$$
f\left(x_{k}+\alpha d_{k}\right)=f_{i_{p}\left(x_{k}+\alpha d_{k}\right)}\left(x_{k}+\alpha d_{k}\right) \in\left[f\left(x_{k}\right)-\frac{\varepsilon}{3}, f\left(x_{k}\right)+\frac{\varepsilon}{3}\right]
$$

Suppose that $j \notin I_{\varepsilon}\left(x_{k}\right)$. So, either $f_{j}\left(x_{k}\right)<f\left(x_{k}\right)-\varepsilon$ or $f_{j}\left(x_{k}\right)>$ $f\left(x_{k}\right)+\varepsilon$. In the first case, by (17), we have that

$$
f_{j}\left(x_{k}+\alpha d_{k}\right)<f\left(x_{k}\right)-\frac{2}{3} \varepsilon
$$


so, by (18),

$$
f_{j}\left(x_{k}+\alpha d_{k}\right)<f\left(x_{k}+\alpha d_{k}\right) .
$$

Analogously, if $f_{j}\left(x_{k}\right)>f\left(x_{k}\right)+\varepsilon$, then

$$
f_{j}\left(x_{k}+\alpha d_{k}\right)>f\left(x_{k}+\alpha d_{k}\right) .
$$

Therefore,

$$
f\left(x_{k}+\alpha d_{k}\right)=f_{j}\left(x_{k}+\alpha d_{k}\right) \text { for some } j \in I_{\varepsilon}\left(x_{k}\right) \text {. }
$$

Let us write

$$
I_{\varepsilon}\left(x_{k}\right)=\left\{j_{1}, \ldots, j_{\nu}\right\}=\left\{j_{1}^{\prime}, \ldots, j_{\nu}^{\prime}\right\},
$$

where

$$
f_{j_{1}}\left(x_{k}\right) \leq \ldots \leq f_{j_{\nu}}\left(x_{k}\right)
$$

and

$$
f_{j_{1}^{\prime}}\left(x_{k}+\alpha d_{k}\right) \leq \ldots \leq f_{j_{\nu}^{\prime}}\left(x_{k}+\alpha d_{k}\right) .
$$

Clearly, there exists $q \in\{1, \ldots, \nu\}$ such that

$$
i_{p}\left(x_{k}\right)=j_{q} .
$$

Now, the indices $j \notin I_{\varepsilon}\left(x_{k}\right)$ such that $f_{j}\left(x_{k}\right)<f\left(x_{k}\right)$ are the same as the indices $j \notin I_{\varepsilon}\left(x_{k}\right)$ such that $f_{j}\left(x_{k}+\alpha d_{k}\right)<f\left(x_{k}\right)$ and, moreover, the indices $j \notin I_{\varepsilon}\left(x_{k}\right)$ such that $f_{j}\left(x_{k}\right)>f\left(x_{k}\right)$ are the same as the indices $j \notin I_{\varepsilon}\left(x_{k}\right)$ such that $f_{j}\left(x_{k}+\alpha d_{k}\right)>f\left(x_{k}\right)$. Then,

$$
i_{p}\left(x_{k}+\alpha d_{k}\right)=j_{q}^{\prime}
$$

Then, by (16) and Lemma 2.1, we have that, when

$$
\alpha \in\left[0, \min \left\{\frac{2 \gamma_{k}(1-\theta)}{L \Delta^{2}}, \frac{\varepsilon}{3 c \Delta}\right\}\right]
$$

we have:

$$
f_{j_{q}^{\prime}}\left(x_{k}+\alpha d_{k}\right) \leq f_{j_{q}}\left(x_{k}\right)+\alpha \theta M_{k}\left(d_{k}\right)
$$

Therefore,

$$
f_{i_{p}\left(x_{k}+\alpha d_{k}\right)}\left(x_{k}+\alpha d_{k}\right) \leq f_{i_{p}\left(x_{k}\right)}\left(x_{k}\right)+\alpha \theta M_{k}\left(d_{k}\right)
$$


So,

$$
f\left(x_{k}+\alpha d_{k}\right) \leq f\left(x_{k}\right)+\alpha \theta M_{k}\left(d_{k}\right) .
$$

Therefore, whenever (19) takes place, the test (13) must hold. This means that a value of $\alpha$ that does not satisfy (13) cannot be smaller than $\min \left\{2 \gamma_{k}(1-\right.$ $\left.\theta) /\left(L \Delta^{2}\right), \varepsilon /(3 c \Delta)\right\}$. So, the accepted $\alpha$ must satisfy:

$$
\alpha_{k} \geq \min \left\{\frac{2 \sigma_{\min } \gamma_{k}(1-\theta)}{L \Delta^{2}}, \frac{\varepsilon \sigma_{\min }}{3 c \Delta}\right\}
$$

as we wanted to prove.

The main convergence result is given in Theorem 2.4. First, we need to prove a simple preparatory lemma.

Lemma 2.2. If $\left\{x_{k}\right\}$ is an infinite sequence generated by Algorithm 2.1, then either

$$
\lim _{k \rightarrow \infty} f\left(x_{k}\right)=-\infty
$$

or

$$
\lim _{k \rightarrow \infty} M_{k}\left(d_{k}\right)=0 .
$$

Proof. If (20) does not hold, then, by (13), we have that

$$
\lim _{k \rightarrow \infty} \alpha_{k} M_{k}\left(d_{k}\right)=0 .
$$

By Theorem 2.3, this implies that (21) takes place.

Theorem 2.4. Suppose that $x_{*} \in \Omega$ is a limit point of a sequence generated by Algorithm 2.1. Then $x_{*}$ is $\varepsilon$-optimal.

Proof. Since $f\left(x_{k+1}\right) \leq f\left(x_{k}\right)$ for all $k=0,1,2, \ldots$ and $x_{*}$ is a limit point of $\left\{x_{k}\right\}$ then $f\left(x_{k}\right) \rightarrow f\left(x_{*}\right)$. Therefore, by Lemma 2.2, (21) takes place. Let $K$ be an infinite sequence of indices such that

$$
\lim _{k \in K} x_{k}=x_{*}
$$

If $x_{*}$ is not $\varepsilon$-optimal, then there exists $\gamma>0$ and $d \in \mathbb{R}^{n}$ such that $x_{*}+d \in \Omega$ and

$$
g_{j}\left(x_{*}\right)^{T} d \leq-\gamma \forall j \in I_{\varepsilon}\left(x_{*}\right)
$$


Without loss of generality we may assume that $\|d\| \leq \Delta / 2$. By continuity, for $k$ large enough, $k \in K$, defining

$$
\hat{d}_{k}=d+x_{*}-x_{k}
$$

we have that $\left\|\hat{d}_{k}\right\|_{\infty} \leq \Delta, x_{k}+\hat{d}_{k} \in \Omega$ and

$$
\lim _{k \in K} \hat{d}_{k}=d
$$

By (21), we have that $\lim _{k \rightarrow \infty} M_{k}\left(\bar{d}_{k}\right)=0$. Therefore, $\liminf _{k \rightarrow \infty} M_{k}\left(\hat{d}_{k}\right) \geq$ 0 . For all $k \in K$ there exists $j \in I_{\varepsilon}\left(x_{k}\right)$ such that $g_{j}\left(x_{k}\right)^{T} \hat{d}_{k}=M_{k}\left(\hat{d}_{k}\right)$. Since $I_{\varepsilon}\left(x_{k}\right)$ is finite, there exists $j$ such that $g_{j}\left(x_{k}\right)^{T} \hat{d}_{k}=M_{k}\left(\hat{d}_{k}\right)$ infinitely many times. Therefore, for that particular $j$,

$$
\liminf _{k \in K} g_{j}\left(x_{k}\right)^{T} \hat{d}_{k}=0 .
$$

Therefore, taking limits,

$$
g_{j}\left(x_{*}\right)^{T} d=0 .
$$

But, for infinitely many indices, since $j \in I_{\varepsilon}\left(x_{k}\right)$,

$$
f\left(x_{k}\right)-\varepsilon \leq f_{j}\left(x_{k}\right) \leq f\left(x_{k}\right)+\varepsilon,
$$

therefore, taking limits,

$$
f\left(x_{*}\right)-\varepsilon \leq f_{j}\left(x_{*}\right) \leq f\left(x_{*}\right)+\varepsilon,
$$

so $j \in I_{\varepsilon}\left(x_{*}\right)$.

Therefore, (24) contradicts (22).

\section{Computing an approximate solution of the sub- problem}

If $n$ is large or $I_{\varepsilon}\left(x_{k}\right)$ contains many elements, computing the exact solution of the (11) can be very costly. In these cases, an approximate solution $d_{k}$ that satisfies (12) can be computed following the procedure described below. To simplify the description we assume that $\operatorname{Int}(\Omega)$, the interior of the convex set $\Omega$, is not empty. Choose $\eta^{\prime} \in(\eta, 1)$. The successive iterates will $x_{k}$ belong to $\operatorname{Int}(\Omega)$. We assume that a procedure exists that computes 
a sequence $\left\{s_{\nu}\right\}$, where $s_{\nu} \in \mathbb{R}^{n}$, such that $s_{\nu} \rightarrow \bar{d}_{k}$ and a sequence of bounds $c_{\nu}$ such that $c_{\nu} \rightarrow M_{k}\left(\bar{d}_{k}\right)$ and

$$
c_{\nu} \leq M_{k}\left(\bar{d}_{k}\right)
$$

for all $\nu=0,1,2, \ldots$

Define, for all $\nu=0,1,2, \ldots$,

$$
\lambda_{\nu}=\min \left\{1, \max \left\{\lambda \geq 0 \mid x_{k}+\lambda s_{\nu} \in \Omega,\left\|\lambda s_{\nu}\right\|_{\infty} \leq \Delta\right\}\right\} .
$$

Since $\Omega$ is convex and $x_{k}$ is interior we have that

$$
\lambda_{\nu} \rightarrow 1
$$

so,

$$
x_{k}+\lambda_{\nu} s_{\nu} \rightarrow x_{k}+\bar{d}_{k}
$$

and

$$
M_{k}\left(\lambda_{\nu} s_{\nu}\right) \rightarrow M_{k}\left(\bar{d}_{k}\right) .
$$

Therefore, taking $\nu$ large enough, we get $\lambda_{\nu}$ such that

$$
M_{k}\left(\lambda_{\nu} s_{\nu}\right) \leq \eta^{\prime} c_{\nu} \leq \eta^{\prime} M_{k}\left(\bar{d}_{k}\right) .
$$

So,

$$
M_{k}\left(\frac{\eta}{\eta^{\prime}} \lambda_{\nu} s_{\nu}\right) \leq \eta M_{k}\left(\bar{d}_{k}\right)
$$

This implies that, taking

$$
d_{k}=\frac{\eta}{\eta^{\prime}} \lambda_{\nu} s_{\nu}
$$

the condition (12) is satisfied, $x_{k}+\alpha d_{k}$ remains interior for all $\alpha \in[0,1]$. So, $x_{k+1}$ is interior and the process can be repeated in the next iteration.

\section{Numerical example}

To illustrate the behavior of the OVO approach, we consider here a fitting problem. We wish to fit the model

$$
y(t, x)=x_{1}+x_{2} t+x_{3} t^{2}+x_{4} t^{3}
$$

to a set of data $\left(t_{i}, y_{i}\right), i=1, \ldots, m$. The function to be minimized is obtained by the set of error functions

$$
f_{i}(x)=\left(y\left(t_{i}, x\right)-y_{i}\right)^{2}
$$


Given the "solution"

$$
x^{*}=\left(x_{1}^{*}, x_{2}^{*}, x_{3}^{*}, x_{4}^{*}\right)=(0,2,-3,1),
$$

we generate the data by

$$
\begin{gathered}
w_{i}=y\left(t_{i}, x^{*}\right), \\
t_{i}=-1+0.1 i, \quad i=0, \ldots, m, m=46, \\
y_{i}=10 \quad \text { if } i=7, \ldots, 16, \\
y_{i}=w_{i}+r_{i}, \text { otherwise }
\end{gathered}
$$

where $r_{i}$ is random between -0.01 and 0.01 . Therefore, $y_{7}, \ldots, y_{26}$ simulate wrong observations, or outliers. In Table 1 we give the data $\left(t_{i}, y_{i}\right)$.

We consider the initial point $x=(-1.0,-2.0,1.0,-1.0)$. Using the Matlab nonlinear least squares solvers POLYFIT and LSQNONLIN (using the bounds given below), we obtained the approximate solutions

$$
x_{P F I T}=(6.4602,2.7072,-7.5418,2.1604)
$$

and

$$
x_{N L L S Q}=(6.4570,2.7048,-7.5364,2.1590),
$$

which are quite far from $x_{*}$. We ran Algorithm 2.1, with the parameters

$$
\Delta=1.0, \quad \theta=0.5, \quad \sigma_{\min }=0.1, \quad \sigma_{\max }=0.9, \quad \eta=1.0, \quad \varepsilon=0.001 .
$$

and the bounds

$$
-10 \leq x_{i} \leq 10, \quad i=1,2,3,4 .
$$

The solutions obtained by the OVO algorithm for different values of $p$ are shown in Table 2, where $p$ define the p-order function, n.iter is the number of iterations, $f o b j$ is the value of the objective function. Observe that the "correct" $p$ should be 36 , because we generated 10 outliers.

The results were coherent and satisfactory. As expected, for $p>36$ the solution given by the algorithm was a point far from $x_{*}$ and the objective function value (OVO function) was large. For $p=27$ and $p=34$ the solution obtained was not a global minimizer of the OVO function. This is not surprising, since the algorithm is guaranteed to obtain only stationary points, therefore it can get nonglobal critical points in some cases. For all the other tested values of $p$ the objective function value obtained was less than 0.05 and the solution obtained was close to $x_{*}$. The example suggests that in real-life situations, when one does not know the number of outliers, different values of $p$ should be tested with different initial points and the solution must be accepted taking into account the values of the OVO function and $p$. 


\begin{tabular}{|c|c|c||c|c|c||c|c|c|}
\hline$i$ & $t_{i}$ & $y_{i}$ & $i$ & $t_{i}$ & $y_{i}$ & $i$ & $t_{i}$ & $y_{i}$ \\
\hline 1 & -1.0000 & -5.8000 & 17 & 0.6000 & 0.5360 & 33 & 2.2000 & 0.3280 \\
\hline 2 & -0.9000 & -5.1590 & 18 & 0.7000 & 0.4730 & 34 & 2.3000 & 1.0970 \\
\hline 3 & -0.8000 & -4.2320 & 19 & 0.8000 & -0.0080 & 35 & 2.4000 & 1.1440 \\
\hline 4 & -0.7000 & -3.4130 & 20 & 0.9000 & 0.2990 & 36 & 2.5000 & 1.6750 \\
\hline 5 & -0.6000 & -2.6960 & 21 & 1.0000 & 0.2000 & 37 & 2.6000 & 2.2960 \\
\hline 6 & -0.5000 & -1.6750 & 22 & 1.1000 & -0.2990 & 38 & 2.7000 & 3.4130 \\
\hline 7 & -0.4000 & 10.0000 & 23 & 1.2000 & 0.0080 & 39 & 2.8000 & 4.2320 \\
\hline 8 & -0.3000 & 10.0000 & 24 & 1.3000 & -0.0730 & 40 & 2.9000 & 5.1590 \\
\hline 9 & -0.2000 & 10.0000 & 25 & 1.4000 & -0.5360 & 41 & 3.0000 & 6.2000 \\
\hline 10 & -0.1000 & 10.0000 & 26 & 1.5000 & -0.5750 & 42 & 3.1000 & 6.9610 \\
\hline 11 & 0 & 10.0000 & 27 & 1.6000 & -0.5840 & 43 & 3.2000 & 8.6480 \\
\hline 12 & 0.1000 & 10.0000 & 28 & 1.7000 & -0.5570 & 44 & 3.3000 & 9.6670 \\
\hline 13 & 0.2000 & 10.0000 & 29 & 1.8000 & -0.4880 & 45 & 3.4000 & 11.2240 \\
\hline 14 & 0.3000 & 10.0000 & 30 & 1.9000 & 0.0290 & 46 & 3.5000 & 12.9250 \\
\hline 15 & 0.4000 & 10.0000 & 31 & 2.0000 & -0.2000 & & & \\
\hline 16 & 0.5000 & 10.0000 & 32 & 2.1000 & 0.0310 & & & \\
\hline
\end{tabular}

Table 1: Data for polynomial fitting with 4 parameters.

\begin{tabular}{|c|c|c|c|c|c|c|}
\hline$p$ & $x_{1}$ & $x_{2}$ & $x_{3}$ & $x_{4}$ & fobj & n.iter \\
\hline 20 & -0.0060 & 1.9892 & -2.9568 & 0.9748 & 0.0404 & 33 \\
\hline 21 & -0.1231 & 1.2809 & -2.4353 & 0.8965 & 0.0369 & 47 \\
\hline 22 & 0.3957 & 2.3052 & -3.6787 & 1.1742 & 0.0297 & 85 \\
\hline 23 & 0.1116 & 1.8346 & -2.9223 & 0.9883 & 0.0401 & 49 \\
\hline 24 & -0.0081 & 2.0035 & -2.9897 & 0.9943 & 0.0405 & 41 \\
\hline 25 & 0.1033 & 1.8469 & -2.9291 & 0.9896 & 0.0402 & 67 \\
\hline 26 & 0.0261 & 1.9557 & -2.9762 & 0.9958 & 0.0403 & 70 \\
\hline 27 & 9.0443 & -7.4778 & -1.4899 & 1.2465 & 2.9491 & 78 \\
\hline 28 & -0.0190 & 2.0336 & -3.0177 & 1.0028 & 0.0405 & 49 \\
\hline 29 & 0.0015 & 1.9917 & -2.9939 & 0.9989 & 0.0407 & 52 \\
\hline 30 & 0.0096 & 1.9841 & -2.9919 & 0.9987 & 0.0403 & 68 \\
\hline 31 & 0.0030 & 2.0024 & -3.0044 & 1.0011 & 0.0407 & 57 \\
\hline 32 & 0.0001 & 2.0030 & -3.0010 & 0.9999 & 0.0408 & 66 \\
\hline 33 & 0.0021 & 2.0007 & -3.0024 & 1.0007 & 0.0407 & 49 \\
\hline 34 & 7.8217 & 5.8390 & -10.0000 & 2.4904 & 14.1827 & 158 \\
\hline 35 & -0.0003 & 2.0004 & -2.9997 & 0.9999 & 0.0403 & 56 \\
\hline 36 & 0.0000 & 2.0003 & -3.0002 & 1.0000 & 0.0403 & 67 \\
\hline 37 & 7.3236 & 1.6767 & -8.3264 & 2.4949 & 13.0175 & 187 \\
\hline 38 & 9.6125 & -9.6561 & -0.5977 & 1.1134 & 10.9439 & 138 \\
\hline 39 & 10.0000 & -6.4565 & -3.4642 & 1.6757 & 15.0919 & 130 \\
\hline 40 & 6.2406 & 2.2166 & -8.3576 & 2.4577 & 16.5412 & 295 \\
\hline 41 & 6.2025 & 2.5359 & -8.6241 & 2.5104 & 17.1385 & 283 \\
\hline 42 & 6.3137 & 1.7981 & -8.3196 & 2.4870 & 19.3482 & 207 \\
\hline 43 & 7.7660 & 2.3983 & -9.6980 & 2.8103 & 24.2539 & 152 \\
\hline
\end{tabular}

Table 2: OVO solution for polynomial fitting with 4 parameters.

\section{Conclusions}

We have introduced the Order-Value Optimization problem, a continuous, nonsmooth and, in general, nonconvex optimization problem that applies to fitting models and, very likely, to decision making. A tentative algorithm, the implementation of which generally relies on Linear Programming subproblems has been defined which seems to work satisfactorily in computer 
generated problems.

The OVO algorithm has a good prospect of dealing with robust fitting problems. Many regression schemes, less sensitive to erroneous data than ordinary least squares, have been introduced in the statistical literature. See, for example, [4], [5] and references therein. A usual approach is to weight the differences by means of a filter that penalizes outliers. This requires some previous knowledge about the data that should be penalized. We think that the OVO algorithm could play the role of a universal filter in the sense that the user needs not know beforehand any kind of qualitative information about the outliers, but only inform the algorithm with a rough estimate of their cardinality in the original sample. The calibration of the model would depend only on the choice of a single 1-dimensional parameter, the whole penalization being done internally by OVO.

On the other hand, the OVO approach can also be used only to detect outliers, leaving the estimation procedure to other algorithm, after the elimination of the detected wrong measurements.

Finally, Order-Value optimization seems to be a challenging optimization problem which generalizes smooth optimization. A promising field of research is to adapt classical optimization algorithms (see, for example, [3]) to the OVO problem. The algorithm presented in this paper is a generalization of the steepest descent method, which, as it is well known, is the more classical algorithm for smooth optimization.

In a parallel research [1] we introduced a smooth reformulation of the OVO problem. This reformulation involves additional auxiliar and dual variables and is related to smooth reformulations of the minimax problem. See, for example, [2]. In general, both the primal method introduced in this paper and the smooth reformulation get the same solutions of the OVO problem. Using the reformulation we get, in theory, convergence of nonlinear programming algorithms to stricter stationary points than the ones defined in this paper. However, the complexity of algorithms based on the reformulation is increased.

Acknowledgement. We are indebted to an anonymous referee whose comments helped us to improve the final version of this paper. 


\section{References}

[1] R. Andreani, C. Dunder and J. M. Martínez, Nonlinear programming reformulation of the Order-Value Optimization problem, Technical Report, Institute of Mathematics, University of Campinas, Brazil, 2001.

[2] G. Di Pillo, L. Grippo and S. Lucidi, A smooth method for the finite minimax problem, Mathematical Programming 60, 187-214, 1993.

[3] R. Fletcher, Practical Methods of Optimization, New York: Wiley, 1988.

[4] Huber, P.J., Robust Statistics, New York: Wiley, 1981.

[5] P. W. Holland and R. E. Welsch, Robust regression using iteratively reweighted least-squares, Communications in Statistics: Theory and Methods, A6, 813-827, 1977. 